\title{
Validez y confiabilidad del cuestionario para medir la adherencia al tratamiento de pacientes con enfermedad cardiovascular
}

\section{RESUMEN}

Objetivo: elaborar y validar un instrumento para medir la adherencia al tratamiento farmacológico y no farmacológico de pacientes con diagnóstico de enfermedad cardiovascular, a partir de un referente teórico sustentado en las conductas de adherencia al tratamiento y la autoeficacia. Materiales y métodos: se detalla el proceso de validez facial, de contenido y de constructo realizado al instrumento, así como las pruebas de confiabilidad. El instrumento fue sometido a evaluación por parte de 6 expertos, 380 adultos de diferentes características sociodemográficas y posteriormente aplicado a 145 pacientes con diagnóstico de enfermedad cardiovascular isquémica. Resultados: se obtuvo un cuestionario con 29 ítems, 6 dimensiones, un alfa de Cronbach de 0,837 y un puntaje entre 29-174, lo que indica que a mayor puntaje mejor adherencia. Conclusiones: se considera un instrumento útil para determinar conductas terapéuticas de adherencia al tratamiento en pacientes con enfermedad cardiovascular isquémica, pues está sustentado en un constructo teórico que se fundamenta, por una parte, en las conductas de adherencia y, por otra, en la autoeficacia como predictor de esta, lo que amplía el abordaje de medición del fenómeno incluida la determinación de las capacidades que perciben los pacientes para poder llevar a cabo un tratamiento.

\section{PALABRAS CLAVE}

Cumplimiento de la medicación; investigación metodológica en enfermería; conductas terapéuticas enfermería cardiovascular; enfermedades cardiovasculares; estudios de validación (Fuente: DeCS, BIREME).

\section{DOI: 10.5294/aqui.2017.17.4.9}

Para citar este artículo / To reference this article / Para citar este artigo Achury-Beltrán LF. Validez y confiabilidad del cuestionario para medir la adherencia al tratamiento de pacientes con enfermedad cardiovascular. Aquichan. 2017; 17(4): 460-471. Doi: 10.5294/aqui.2017.17.4.9 


\title{
Validity and Reliability of a Questionnaire to Measure Adherence to Treatment by Patients with Cardiovascular Disease
}

\begin{abstract}
Objective: Develop and validate an instrument to measure adherence to pharmacological and non-pharmacological treatment on the part of patients diagnosed with cardiovascular disease, based on a theoretical reference supported by treatment adherence behavior and self-efficacy. Materials and methods: The process of facial, content and construct validity performed on the instrument is outlined in detail, as are the reliability tests. The instrument was subject to evaluation by six experts and 380 adults with different sociodemographic characteristics, before being applied to 145 patients who had been diagnosed with ischemic cardiovascular disease. Results: A questionnaire with 29 items, 6 dimensions, a Cronbach's alpha of 0.837 and a score of 29-174 was obtained, indicating that the higher the score, the better the adherence. Conclusions: It is considered a useful instrument to determine therapeutic behavior in terms of adherence to treatment among patients with ischemic cardiovascular disease, since it is based on a theoretical construct that is founded on adherence behavior, on the one hand, and self-efficacy, on the other. The latter is a predictor of adherence behavior. This broadens the approach to measuring the phenomenon, including determining the abilities or capacity perceived by patients in order to complete a treatment.
\end{abstract}

\section{KEYWORDS}

Compliance with medication; methodological research in nursing; therapeutic behavior; cardiovascular nursing; cardiovascular diseases; validation studies (Source: DeCS, BIREME). 


\section{Validade e confiabilidade do questionário para medir a adesão ao tratamento de pacientes com doença cardiovascular}

\section{RESUMO}

Objetivo: elaborar e validar um instrumento para medir a adesão ao tratamento farmacológico e não farmacológico de pacientes com diagnóstico de doença cardiovascular, a partir de um referencial teórico apoiado nos comportamentos de adesão ao tratamento e na autoeficácia. Materiais e métodos: detalha-se o processo de validade facial, de conteúdo e de construto realizado ao instrumento, bem como os testes de confiabilidade. 0 instrumento foi submetido à avaliação por parte de 6 especialistas, 380 adultos de diferentes características sociodemográficas e, em seguida, aplicado a 145 pacientes com diagnóstico de doença cardiovascular isquêmica. Resultados: obteve-se um questionário com 29 itens, 6 dimensões, um alfa de Cronbach de 0.837 e uma pontuação entre 29-174, o que indica que quanto maior a pontuação, melhor a adesão. Conclusões: considera-se um instrumento útil para determinar comportamentos terapêuticos de adesão ao tratamento em pacientes com doença cardiovascular isquêmica, pois está sustentado num construto teórico que se fundamenta, por um lado, nos comportamentos de adesão e, por outro, na autoeficácia como preditor desta, o que amplia a abordagem de medição do fenômeno incluída a determinação das capacidades que percebem os pacientes para poder realizar um tratamento.

\section{PALAVRAS-CHAVE}

Comportamentos terapêuticos; cumprimento da medição; doenças cardiovasculares; enfermagem cardiovascular; estudos de validação; pesquisa metodológica em enfermagem (Fonte: DeCS, Bireme). 


\section{Introducción}

Las enfermedades cardiovasculares hacen parte del grupo de las enfermedades no transmisibles, las cuales, según la Organización Mundial de la Salud (OMS), son las principales causas de morbilidad y mortalidad a nivel mundial. De acuerdo con las estadísticas de la OMS, durante el 2012 se registraron 38 millones de muertes por enfermedades no transmisibles, de las cuales 17,5 millones correspondieron a enfermedad cardiovascular (1). Por su parte, la American Heart Association reportó que en el 2013 la tasa de muertes atribuibles a enfermedades cardiovasculares fue de 222,9 por/100.000 americanos y, a pesar de que durante el periodo comprendido entre 2003 y 2013 la tasa se redujo en un $28,8 \%$, en el 2013 las enfermedades cardiovasculares todavía representaban el $30,8 \%$ de la mortalidad (2).

En la actualidad se estima que 85,6 millones de adultos americanos padecen de mínimo un tipo de enfermedad cardiovascular, de los cuales 15,5 millones corresponden a enfermedad coronaria. Específicamente en la población latinoamericana, 7,8\% padece de enfermedad cardiovascular y el $4,9 \%$ de enfermedad coronaria (2). En Colombia, el panorama no es muy distinto, pues la enfermedad cardiovascular ocupa también el primer lugar como causa de mortalidad con 8.403 muertes derivadas de enfermedad isquémica del corazón, 1.899 de enfermedades hipertensivas, 1.002 de otras formas de enfermedad cardiaca, 573 de insuficiencia cardiaca y 3.741 de enfermedad cerebrovascular (3).

Se ha descrito cómo una adecuada adherencia al tratamiento farmacológico y no farmacológico se convierte en un aspecto imprescindible del manejo de la enfermedad cardiovascular, puesto que asegura su adecuado control, una menor tasa de descompensaciones y un incremento en la calidad de vida como desenlaces primarios (4). Por esta razón, la medición de la adherencia se convierte en un aspecto esencial, ya que su identificación le permite al profesional de enfermería detectar las necesidades concretas de los pacientes $y$, a partir de allí, generar diversas estrategias con el fin de intervenir de forma oportuna y eficaz para mejorar la calidad de vida, reducir los reingresos hospitalarios, favorecer la reincorporación a la vida laboral, familiar y social de los pacientes, y reducir las complicaciones que pueden generarse de un manejo subóptimo de la situación de salud $(5,6)$.

Con el propósito de determinar la adherencia a los tratamientos de la enfermedad, algunos autores han implementado métodos objetivos como el recuento de tabletas, las tasas de asistencia a los programas, la obtención de resultados terapéuticos y las mediciones de fármacos cuando ello es posible $(7,8)$, sin embargo, dada la complejidad de dichos controles, otros autores han elaborado mediciones subjetivas que incluyen instrumentos que determinan la presencia de factores que influyen en la adherencia terapéutica, lo que permite pronosticarla pero no determinarla de forma concreta (9-13).

Adicionalmente, a pesar de la diversidad de técnicas de medición existentes, hay evidencia de que la adherencia está robustamente influenciada por la motivación, ya que esta guarda una fuerte relación con la importancia que el paciente le asigna al cumplimento de los tratamientos y, además, a la confianza que tiene en poder seguirlos (14), aspectos claves de la autoeficacia. Al respecto, las teorías de Albert Bandura y Bárbara Resnick, citadas por Resnick (15), abordan el concepto de la motivación en la medida en que explican cómo la autoeficacia influye en la generación de comportamientos motivados que finalmente son capaces de generar cambios de conductas en los pacientes y favorecer la adherencia terapéutica. Por ese motivo, se hace necesario el diseño y la validación de un instrumento que además de evaluar las conductas que demuestran la adherencia de los pacientes con diagnóstico de enfermedad cardiovascular isquémica en términos del seguimiento a las recomendaciones de tratamiento farmacológico y no farmacológico, también valore la autoeficacia como un predictor de la motivación y, con ello, de la capacidad de los pacientes para llevar a cabo las acciones requeridas como parte del manejo.

Con este fin, el instrumento diseñado evalúa, por una parte, las conductas de los pacientes en términos de la adherencia al tratamiento no farmacológico de la enfermedad cardiovascular isquémica, dentro de las que se incluyen la dieta, la actividad física, el control de factores riesgo como el consumo de tabaco 0 alcohol y el manejo del estrés (16-18), y por otra, el tratamiento farmacológico que hace referencia al consumo de los medicamentos prescritos en las dosis y las frecuencias indicadas. Además, incluye ítems que evalúan la autoeficacia como un elemento predictor de la adherencia, en la medida en que indagan acerca de la percepción de los pacientes para llevar a cabo diferentes acciones requeridas como parte del manejo de la enfermedad.

\section{Objetivo}

Elaborar y validar un instrumento para medir la adherencia al tratamiento farmacológico y no farmacológico de pacientes con enfermedad cardiovascular isquémica, a partir de un referente 
teórico sustentado en las conductas de adherencia al tratamiento y la autoeficacia como predictor de esta.

\section{Materiales y métodos}

El estudio se desarrolló bajo un enfoque metodológico con una perspectiva psicométrica que contempló la elaboración y validación del instrumento de medición de adherencia denominado "Cuestionario para medir la adherencia al tratamiento en pacientes con enfermedad cardiovascular".

La muestra estuvo conformada por tres grupos: 6 expertos quienes realizaron el proceso de validez facial y de contenido del instrumento, 380 adultos de diferentes características sociodemográficas para determinar la validez facial a través del índice de comprensibilidad y, finalmente, 145 pacientes con enfermedad cardiovascular isquémica para determinar la validez de constructo y la confiabilidad del cuestionario.

Para la inclusión en el estudio, los expertos y demás participantes debieron ser mayores de 18 años, demostrar capacidad mental para responder o evaluar el instrumento y aceptar de forma voluntaria su participación en la investigación, aspectos que se enmarcan en la Resolución 008430 de 1993 del entonces Ministerio de Salud de la República de Colombia, y las pautas éticas del Consejo de Organizaciones de Ciencias Médicas y la OMS. Adicionalmente, el estudio fue avalado por los comités de ética e investigación de la Universidad Nacional de Colombia y de la institución en donde se desarrolló el estudio según las actas 06 del 14 de abril de 2014 y 7-2014 del 24 de abril del mismo año.

\section{Procedimiento}

El desarrollo de la investigación se produjo en dos fases:

- La primera fase correspondió a la revisión de la literatura y la definición del constructo para la elaboración de los ítems del instrumento.

- La segunda fase involucró la realización de la validez facial, de contenido y de constructo, así como el cálculo de la confiabilidad de la versión final del instrumento.

Para la primera fase de revisión de la literatura, el investigador realizó una búsqueda de artículos publicados durante el periodo 2000-2015 en español, inglés y portugués, en las bases de datos Medline, ProQuest, Ovid y Science Direct, bajo los descriptores "enfermería", "enfermedad cardiovascular", "enfermedad coronaria", "adherencia terapéutica", "infarto agudo de miocardio" y "rehabilitación cardiaca" con los conectores booleanos AND y OR, con el fin de determinar las conductas que deben contemplarse dentro de la evaluación de la adherencia al tratamiento farmacológico y no farmacológico de los pacientes con enfermedad cardiovascular isquémica, así como instrumentos de medición de adherencia; dicha búsqueda, además de facilitar la definición del constructo, dio lugar a la identificación de instrumentos tales como la "Escala de adherencia terapéutica para pacientes con enfermedades crónicas basada en comportamientos explícitos" (19), el "Cuestionario de adherencia al tratamiento para la hipertensión arterial (CAT_HTA)" (20) y el "Cuestionario para la evaluación de la adherencia terapéutica de Martin-Bayarre-Grau" (10).

A partir de dicha revisión de la literatura y de la definición del constructo de la adherencia al tratamiento en el paciente con enfermedad cardiovascular isquémica, se construyó inicialmente un instrumento de 26 ítems, de los cuales dos evaluaban la ingesta de medicamentos, tres la ingesta de alimentos, tres la realización de actividad física, cuatro el manejo de factores de riesgo, seis el seguimiento y control de la enfermedad y, finalmente, ocho los resultados de autoeficacia.

Una vez el proyecto de investigación fue sometido a evaluación y aprobación por parte de los comités de ética e investigación de la Universidad Nacional de Colombia y la institución hospitalaria seleccionada, el investigador eligió seis expertos que cumplían los criterios de Skjong y Wentworth (21), dentro de los que se incluyen: experiencia en la realización de juicios y decisiones basada en evidencia o experticia, reputación en la comunidad, disponibilidad y motivación para participar e imparcialidad, y posteriormente se diseñó un formato para realizar el proceso de validez facial y de contenido.

Para la validez de contenido se le solicitó a los expertos evaluar cada uno de los ítems en términos de "esencial", "útil pero no esencial" y "no necesario" para, posteriormente, determinar la razón de validez de contenido (CVR') por medio de la fórmula $n_{e} / N$, en donde $n_{e}$ corresponde a la cantidad de expertos que calificaron el ítem como esencial y $\mathrm{N}$ el total de expertos que evaluaron el ítem. Se consideró como límite inferior de aceptabilidad una CVR' de 0,67, tal como lo estipula Tristán cuando la evaluación es realizada con seis expertos (22). 
Adicionalmente, la validez facial fue realizada tanto por los seis expertos a quienes se les pidió evaluar los ítems en términos de comprensión, claridad y precisión para calcular el índice de concordancia simple y el índice de kappa de Fleiss, como por 380 individuos mayores de 18 años de diferentes características sociodemográficas como edad, estrato socioeconómico y escolaridad, quienes calificaron los ítems bajo los criterios de "se entiende", "se entiende parcialmente" o "no se entiende", lo que permitió determinar el grado de comprensión del instrumento a partir del cálculo del índice de comprensibilidad neta por ítem.

El índice de kappa de Fleiss fue interpretado a partir de los puntos de corte establecidos por Landis y Koch en donde $<0=$ pobre acuerdo; $0,01-0,2=$ acuerdo débil, $0,21-0,40=$ acuerdo aceptable; $0,41-0,60=$ acuerdo moderado; $0,61-0,8=$ acuerdo sustancial y $0,81-1=$ acuerdo casi perfecto (23).

Por otra parte, en el índice de comprensibilidad se codificaron las respuestas (se entiende $=2$, se entiende parcialmente $=1 \mathrm{y}$ no se entiende $=0$ ) para posteriormente establecer el porcentaje de comprensibilidad de cada uno de los ítems, de manera que se estableció que un resultado menor al $90 \%$ implicaba una restructuración a fondo del ítem, entre $90-95 \%$ un ajuste menor y mayor al $95 \%$ no requería modificación alguna.

Posteriormente, se realizó la validez de constructo. Para ello, previa firma del consentimiento informado, se aplicó la última versión del instrumento a 145 pacientes con enfermedad cardiovascular isquémica con el objetivo de realizar un análisis factorial exploratorio con el método de extracción por componentes principales y rotación Varimax, utilizando un criterio de extracción de 1,41 como lo estipula Gorsuch, citado por Campo, Herazo y Oviedo (24), y el programa estadístico SPSS versión 22 con licencia para la Universidad Nacional de Colombia. Por último, se determinó la consistencia interna del instrumento a partir del cálculo del alfa de Cronbach.

\section{Resultados}

A continuación se presentan los resultados de la validez facial, de contenido y de constructo, así como la consistencia interna del instrumento denominado "Cuestionario para medir la adherencia al tratamiento en pacientes con enfermedad cardiovascular".

\section{Validez de contenido}

A partir de la evaluación de los ítems por parte de los expertos se aplicó la fórmula de razón de validez de contenido (CVR') $y$, con base en el límite inferior de aceptabilidad que establece Tristán (22), se decidió la eliminación de dos ítems ("como solo los alimentos que el personal de salud me indica", "destino diariamente un tiempo específico para realizar ejercicio") (tabla 1). De forma adicional, por sugerencia de los expertos, se incluyeron dos ítems que se consideraron relevantes para determinar el fenómeno de la adherencia en la población de estudio ("evito en mi alimentación el consumo de harinas y azúcares", "consumo alimentos bajos en sal"); quedó así un instrumento de 26 ítems nuevamente.

Tabla 1. Razón de validez de contenido de los ítems del "Cuestionario para medir la adherencia al tratamiento en pacientes con enfermedad cardiovascular"

\begin{tabular}{|c|c|}
\hline Ítem & CVR' \\
\hline 1 & 1,00 \\
\hline 2 & 1,00 \\
\hline 3 & 1,00 \\
\hline 4 & 0,33 \\
\hline 5 & 1,00 \\
\hline 6 & 0,83 \\
\hline 7 & 0,67 \\
\hline 8 & 0,83 \\
\hline 9 & 0,83 \\
\hline 10 & 1,00 \\
\hline 11 & 0,83 \\
\hline 12 & 1,00 \\
\hline 13 & 1,00 \\
\hline
\end{tabular}

\begin{tabular}{|c|c|}
\hline Ítem & CVR' \\
\hline 14 & 1,00 \\
\hline 15 & 1,00 \\
\hline 16 & 1,00 \\
\hline 17 & 0,83 \\
\hline 18 & 1,00 \\
\hline 19 & 1,00 \\
\hline 20 & 1,00 \\
\hline 21 & 0,83 \\
\hline 22 & 0,83 \\
\hline 23 & 1,00 \\
\hline 24 & 1,00 \\
\hline 25 & 1,00 \\
\hline 26 & 1,00 \\
\hline
\end{tabular}

Fuente: elaboración propia.

\section{Validez facial}

A partir de la evaluación del instrumento por parte de los seis expertos se realizaron los ajustes correspondientes acatando las recomendaciones, y se calculó el índice de concordancia simple para cada uno de los ítems en los aspectos de compren- 
sión, claridad y precisión. Los resultados sustentaron la eliminación de los ítems 4 y 7, tal como lo había revelado el proceso de validez de contenido.

Por otra parte, como se muestra en la tabla 2, el índice de kappa de Fleiss general y por dimensiones, a partir de los criterios de Landis y Koch señalados por Cerda y Villarroel (23), reveló un acuerdo sustancial en todas las dimensiones en el aspecto de comprensión; un acuerdo sustancial en términos de claridad en todas las dimensiones excepto en la de factores de riesgo, la cual mostró un acuerdo moderado, y para el aspecto de precisión, un acuerdo sustancial en las dimensiones de ingesta de medicamentos, seguimiento / control y resultados de autoeficacia; un acuerdo moderado en realización de actividad física y factores de riesgo y, finalmente, un acuerdo aceptable en la dimensión de ingesta de alimentos. De forma general, el instrumento reveló un acuerdo sustancial para los aspectos de compresión y claridad, y un acuerdo moderado para el aspecto de precisión, lo que llevó a sacar los dos ítems que correspondían a las preguntas de frecuencia de la escala Likert y convertirlos en preguntas de selección múltiple.

Tabla 2. Índice de kappa de Fleiss general y por dimensiones del cuestionario de acuerdo con los aspectos de compresión, claridad y precisión.

\begin{tabular}{|l|c|l|l|l|l|l|}
\hline \multirow{2}{*}{ Dimensión } & \multicolumn{5}{|c|}{ Kappa de Fleiss } \\
\cline { 2 - 7 } & \multicolumn{2}{|c|}{ Comprensión } & \multicolumn{2}{|c|}{ Claridad } & \multicolumn{2}{c|}{ Precisión } \\
\hline $\begin{array}{l}\text { Ingesta de } \\
\text { medicamentos }\end{array}$ & 0,77 & Sustancial & 0,77 & Sustancial & 0,65 & Sustancial \\
\hline $\begin{array}{l}\text { Ingesta de } \\
\text { alimentos }\end{array}$ & 0,76 & Sustancial & 0,62 & Sustancial & 0,28 & Aceptable \\
\hline $\begin{array}{l}\text { Realización } \\
\text { de actividad } \\
\text { física }\end{array}$ & 0,76 & Sustancial & 0,62 & Sustancial & 0,48 & Moderado \\
\hline $\begin{array}{l}\text { Factores de } \\
\text { riesgo }\end{array}$ & 0,66 & Sustancial & 0,59 & Moderado & 0,46 & Moderado \\
\hline $\begin{array}{l}\text { Seguimiento / } \\
\text { control }\end{array}$ & 0,77 & Sustancial & 0,77 & Sustancial & 0,69 & Sustancial \\
\hline $\begin{array}{l}\text { Resultados de } \\
\text { autoeficacia }\end{array}$ & 0,71 & Sustancial & 0,71 & Sustancial & 0,66 & Sustancial \\
\hline General & 0,73 & Sustancial & 0,69 & Sustancial & 0,57 & Moderado \\
\hline
\end{tabular}

Fuente: elaboración propia.

Posteriormente, el instrumento fue sometido a evaluación por parte de 380 individuos de diferentes edades, estratos socioeco- nómicos y escolaridades, con el fin de determinar la comprensibilidad neta de cada uno de los 26 ítems. A pesar de que la totalidad de los ítems del instrumento reportó una comprensibilidad neta superior al 95\%, con una mínima reportada de 96,05\%, se tuvieron en cuenta las apreciaciones de algunos de los participantes lo que llevó a dividir la pregunta 16 en 3 ítems ("por alguna razón suspendo mis medicamentos sin la indicación del personal de salud", "por alguna razón dejo de seguir las recomendaciones de la alimentación dadas por el personal de salud", "por alguna razón suspendo el ejercicio físico sin la indicación del personal de salud"). Con ello, se obtuvo un instrumento de 29 ítems de los cuales 27 tienen una opción de respuesta tipo Likert (nunca, casi nunca, pocas veces, frecuentemente, casi siempre y siempre), y los 2 ítems restantes respuestas de selección múltiple.

Para las preguntas 1, 2, 4, 5, 6, 7, 9, 11-17, 23-27, las opciones de respuesta tienen las siguientes puntuaciones: nunca $=1$, casi nunca $=2$, pocas veces $=3$, frecuentemente $=4$, casi siempre $=5$ y siempre $=6$. Para los ítems 3, 8, 10, 18-22: nunca $=6$, casi nunca $=5$, pocas veces $=4$, frecuentemente $=3$, casi siempre $=2$ y siempre $=1$. Finalmente, para las preguntas de selección múltiple (28 y 29), las puntuaciones son las siguientes: varias veces al día $=1$, una vez al día $=2$, varios días a la semana $=3$, una vez a la semana $=4$, de vez en cuando $=5$ y no consumo bebidas alcohólicas $/$ fumo $=6$. En este sentido, el instrumento tiene una puntuación mínima de 29 y máxima de 174, de manera que un puntaje mayor indica una mejor adherencia al tratamiento.

\section{Validez de constructo}

La validez de constructo del instrumento final fue evaluada a partir de un análisis factorial exploratorio con el método de extracción por componentes principales y rotación Varimax, el cual arrojó una estructura de 6 componentes que dan cuenta del $57,36 \%$ de la varianza explicada, lo que identifica las dimensiones que establecen las relaciones entre los ítems que componen el instrumento (25) (tabla 3). Es importante mencionar que la matriz de componentes principales rotada que se obtuvo del procedimiento estadístico dejó ver una agrupación de los ítems que no coincide exactamente con la definida previamente en el instrumento; sin embargo, es posible redefinirlas en razón de que las expectativas de autoeficacia están presentes en todos los tratamientos de los pacientes con enfermedad cardiovascular. 
Tabla 3. Matriz rotada de componentes principales de los ítems del "Cuestionario para medir la adherencia al tratamiento de pacientes con enfermedad cardiovascular"

\begin{tabular}{|c|c|c|c|c|c|c|}
\hline \multirow{2}{*}{ Ítem s } & \multicolumn{5}{|c|}{ Componente } \\
\hline & $\mathbf{1}$ & $\mathbf{2}$ & $\mathbf{3}$ & $\mathbf{4}$ & $\mathbf{5}$ & $\mathbf{6}$ \\
\hline Ítem 1 & & 0,749 & & & & \\
\hline Ítem 2 & & 0,799 & & & & \\
\hline Ítem 3 & & 0,701 & & & & \\
\hline Ítem 4 & & 0,521 & & & & \\
\hline Ítem 5 & 0,545 & & & & & \\
\hline Ítem 6 & 0,437 & & & & & \\
\hline Ítem 7 & 0,467 & & & & & \\
\hline Ítem 8 & & 0,502 & & & & \\
\hline Ítem 9 & & & & & & 0,784 \\
\hline Ítem 10 & & & & & & 0,414 \\
\hline Ítem 11 & & & & & & 0,632 \\
\hline Ítem 12 & & & & & 0,750 & \\
\hline Ítem 13 & & & & & 0,815 & \\
\hline Ítem 14 & & & & 0,759 & & \\
\hline Ítem 15 & & & & 0,608 & & \\
\hline Ítem 16 & & & & 0,647 & & \\
\hline Ítem 17 & 0,447 & & & & & \\
\hline Ítem 18 & & & 0,772 & & & \\
\hline Ítem 19 & & & 0,762 & & & \\
\hline Ítem 20 & & & 0,815 & & & \\
\hline Ítem 21 & & 0,610 & & & & \\
\hline Ítem 22 & & & & & & 0,423 \\
\hline Ítem 23 & & & & & 0,557 & \\
\hline Ítem 24 & 0,676 & & & & & \\
\hline Ítem 25 & 0,702 & & & & & \\
\hline Ítem 26 & 0,719 & & & & & \\
\hline Ítem 27 & 0,749 & & & & & \\
\hline Ítem 28 & & & & 0,422 & & \\
\hline Ítem 29 & & & & 0,352 & & \\
\hline
\end{tabular}

Fuente: elaboración propia.
De este modo, las dimensiones se denominaron de la siguiente forma: expectativas y conductas de autoeficacia, seguimiento a las indicaciones del personal de salud en relación con la ingesta de medicamentos y alimentos, necesidad de apoyo para la adherencia, conductas esperadas de adherencia a largo plazo, manejo de situaciones tensionantes y realización de actividad física.

\section{Confiabilidad: consistencia interna}

El alfa de Cronbach para el instrumento general reportó 0,837 , lo cual indica un buen nivel de fiabilidad y con ello de correlación entre los ítems del cuestionario. Adicionalmente, se puede evidenciar que el alfa de Cronbach no presenta variaciones significativas cuando alguno de los ítems del cuestionario es eliminado, de manera que este fluctúa entre 0,825 y 0,840 (tabla 4).

Por otra parte, el cálculo del alfa de Cronbach para cada una de las dimensiones arrojadas por el análisis factorial demostró unos valores buenos de fiabilidad tanto para la dimensión de conductas y expectativas de autoeficacia como para la dimensión de necesidad de apoyo para la adherencia; un valor aceptable para la dimensión de seguimiento a las indicaciones del personal de salud en relación con la ingesta de medicamentos y alimentos; una fiabilidad débil para las dimensiones de realización de actividad física y manejo de las situaciones tensionantes y, finalmente, un valor pobre para la dimensión de conductas esperadas de adherencia a la largo plazo.

\section{Discusión}

La adherencia al tratamiento es un fenómeno multicausal que se ve influenciado por un sinnúmero de condiciones que guardan relación con los factores derivados de la enfermedad, el tratamiento, el paciente, las características socioeconómicas y del equipo de salud; sin embargo, la literatura reporta que abordar el fenómeno de adherencia al tratamiento en la enfermedad cardiovascular implica, por una parte, conocer el cumplimiento de aspectos tanto farmacológicos como no farmacológicos dentro de los que se incluyen el consumo de medicamentos, la dieta, la actividad física, la asistencia a controles médicos y el manejo de los factores de riesgo, y por otra, determinar la autoeficacia como un aspecto relevante que influye en la motivación y adopción de conductas promotoras de salud.

Si bien es importante mencionar la existencia de algunos instrumentos relacionados con la medición de la adherencia al 
Tabla 4. Fiabilidad del "Cuestionario para medir la adherencia al tratamiento de pacientes con enfermedad cardiovascular"

\begin{tabular}{|c|c|c|}
\hline No. & Ítem & $\begin{array}{l}\text { Alfa de C. si } \\
\text { se suprime } \\
\text { el elemento }\end{array}$ \\
\hline 1 & Tomo mis medicamentos en el horario establecido & 0,830 \\
\hline 2 & Tomo diariamente todos mis medicamentos de acuerdo con la orden médica & 0,830 \\
\hline 3 & Por alguna razón suspendo mis medicamentos sin la indicación del personal de salud & 0,833 \\
\hline 4 & Sigo las indicaciones del personal de salud en relación con mi alimentación & 0,825 \\
\hline 5 & Evito en mi alimentación el consumo de grasa (pollo con piel, fritos, alimentos de paquete, leche entera, alimentos precocidos) & 0,832 \\
\hline 6 & Evito en mi alimentación el consumo de harinas y azúcares & 0,828 \\
\hline 7 & Consumo alimentos bajos en sal & 0,835 \\
\hline 8 & Por alguna razón dejo de seguir las recomendaciones de la alimentación dadas por el personal de salud & 0,829 \\
\hline 9 & Realizo el ejercicio físico indicado por el personal de salud & 0,831 \\
\hline 10 & Por alguna razón suspendo el ejercicio físico sin la indicación del personal de salud & 0,832 \\
\hline 11 & Realizo ejercicio físico al menos 3 veces por semana, con una duración mínima de 30 minutos en cada oportunidad & 0,832 \\
\hline 12 & Realizo actividades que me ayuden a manejar el estrés o las situaciones tensionantes & 0,834 \\
\hline 13 & Cuando estoy angustiado hago algo que me ayude a sentirme mejor & 0,831 \\
\hline 14 & Asisto a las consultas médicas programadas & 0,830 \\
\hline 15 & Asisto a los controles de enfermería programados & 0,830 \\
\hline 16 & Me realizo los exámenes de laboratorio y otros exámenes en los periodos que los profesionales de la salud me indican & 0,832 \\
\hline 17 & $\begin{array}{l}\text { Estoy atento a cualquier signo o síntoma que demuestre un empeoramiento en mi estado de salud como: dolor en el pecho, } \\
\text { presión arterial alta o baja, palpitaciones, ahogo o fatiga, inflamación de brazos y piernas }\end{array}$ & 0,830 \\
\hline 18 & Necesito que otras personas me recuerden que debo tomar mis medicamentos & 0,831 \\
\hline 19 & Necesito que otras personas me recuerden que debo seguir la alimentación ordenada por el personal de salud & 0,832 \\
\hline 20 & Necesito que otras personas me recuerden que debo hacer el ejercicio físico ordenado por el personal de salud & 0,838 \\
\hline 21 & Si en poco tiempo no presento mejoría en mi salud, suspendo mis medicamentos & 0,832 \\
\hline 22 & Si en poco tiempo no presento mejoría en mi salud, suspendo el ejercicio físico & 0,835 \\
\hline 23 & Puedo manejar las situaciones tensionantes o que me producen estrés & 0,834 \\
\hline 24 & Soy capaz de cambiar los comportamientos dañinos para mi salud & 0,831 \\
\hline 25 & Cumplo el tratamiento de mi enfermedad aunque me parezca complicado & 0,829 \\
\hline 26 & Hago lo que está a mi alcance para mejorarme cuando estoy enfermo & 0,832 \\
\hline 27 & Soy capaz de seguir las indicaciones que me da el personal de salud & 0,831 \\
\hline 28 & ¿Con qué frecuencia consume bebidas alcohólicas? & 0,836 \\
\hline 29 & ¿Con qué frecuencia fuma? & 0,840 \\
\hline \multicolumn{2}{|c|}{ Alfa de Cronbach general } & 0,837 \\
\hline
\end{tabular}


tratamiento, como los elaborados por Ortiz (12) y Contreras et al. (13), se debe destacar que ninguno de ellos contempla explícitamente las conductas de adherencia y la autoeficacia como un factor predictor de la motivación y del cambio, lo que lleva al diseño y elaboración de un instrumento basado en los lineamientos de Streiner, Norman y Cairney (26), quienes consideran dentro del procedimiento la definición del constructo, la elaboración de los ítems y su escala de medida, la validez facial, de contenido y de constructo, así como la confiabilidad del mismo.

La validez de contenido de un instrumento determina qué tan apropiados son los ítems en relación con el constructo que se desea medir (27), y si bien como resultado de esta fase se determinó la eliminación de dos ítems, esto guarda relación con la presencia en el instrumento de otras preguntas que permiten identificar la adherencia de los pacientes a la dieta y al ejercicio, por lo que los resultados demuestran la pertinencia de los tópicos contemplados en el instrumento y el carácter multicausal de la adherencia al tratamiento, tal como lo evidencian Ortego (28) y Silva et al. (29), quienes consideran que en la medición de este fenómeno deben contemplarse el seguimiento de las indicaciones relacionadas con la dieta y el ejercicio, la evitación de conductas de riesgo, el cumplimiento de las citas programadas, el consumo correcto de la medicación y otros factores dentro de los cuales cobra especial relevancia la motivación.

Por su parte, la validez facial determina si en apariencia un instrumento mide lo que desea medir; para ello, se contempla el uso del índice de kappa de Fleiss, el cual es una medida estadística que permite determinar la concordancia interobservador, en este caso, el acuerdo entre los expertos con respecto a la evaluación del instrumento en mención (23). Es así como el proceso de validez facial realizado deja ver tanto la concordancia sustancial y moderada interobservador de los expertos, como la comprensión de cada una de las preguntas contempladas como parte de la evaluación de la adherencia al tratamiento del paciente con diagnóstico de enfermedad cardiovascular, lo que demuestra que este fenómeno debe ser evaluado a partir de la indagación de diferentes aspectos tal y como lo mencionan Ortego (28), Veliz, Medoza y Barrigac (30) y Martin, Grau y Espinosa (31).

La validez de constructo determina el grado en el que un instrumento mide la dimensión evaluativa para la que fue diseñado, es decir, la relación del instrumento con la teoría y con su conceptualización (32); a partir de esta se obtuvieron las nuevas di- mensiones del instrumento, las cuales pueden articularse con los resultados de investigaciones existentes. Olivari y Urra-Medina (33), por su parte, consideran que la autoeficacia influye sobre la percepción que tienen los pacientes acerca de sus capacidades y de los objetivos que pueden cumplirse tras la implementación de los tratamientos, lo que incrementa la probabilidad de llevar a cabo cambios de comportamiento y aumenta la adherencia, por tanto, es posible considerar que la autoeficacia es un concepto que resulta ser transversal a la mayoría de las conductas requeridas como parte del manejo, incluidos, entre otros, la realización de la actividad física tal como lo reporta Reigal y Cidera (34). Por su parte, otros autores como Silva et al. (29), Berciano y Ordovás (35), y Perkl et al. (36) destacan la importancia del consumo de medicamentos, el seguimiento de la dieta, la realización de actividad física, la asistencia a controles médicos y la toma de paraclínicos ordenados como parte indispensable del tratamiento, por lo que dichos aspectos deben ser incluidos dentro de los instrumentos que tienen como finalidad determinar la adherencia.

Finalmente, el cálculo del alfa de Cronbach permite determinar la correlación de los ítems dentro del cuestionario y valora la manera como cada uno de ellos estima las mismas características (37), por tanto, los valores obtenidos dejan ver la buena confiabilidad del instrumento en general y la homogeneidad de los enunciados del mismo, así como la correlación existente entre ellos, tal como lo establecen Carvajal et al. (38), lo que lo hace un instrumento prometedor en la evaluación de la adherencia al tratamiento en pacientes con diagnóstico de enfermedad cardiovascular.

\section{Conclusiones}

El estudio metodológico de enfoque psicométrico dio lugar a un instrumento con 6 dimensiones y 29 ítems, los cuales arrojan un puntaje que oscila entre 29 y 174 puntos, en donde un mayor puntaje revela una mayor adherencia al tratamiento. Adicionalmente, se demostró de forma global un buen nivel de fiabilidad, con un alfa de Cronbach de 0,837 , que lo convierte en un instrumento prometedor para determinar conductas de adherencia al tratamiento en pacientes con diagnóstico de enfermedad cardiovascular isquémica.

El cuestionario para medir la adherencia al tratamiento en pacientes con enfermedad cardiovascular está sustentado en un constructo teórico que se fundamenta, por una parte, en las conductas de adherencia y, por otra, en la autoeficacia como predic- 
tor de esta, de manera que se amplía el abordaje de medición del fenómeno incluida la determinación de las capacidades que perciben los pacientes para poder llevar a cabo una determinada acción del tratamiento.

Dado que este instrumento permite determinar tanto las conductas de adherencia al tratamiento como las expectativas y conductas de autoeficacia, el profesional de enfermería podrá no solo identificar la adherencia de los pacientes, sino también diseñar e implementar estrategias que le permitan, desde diferentes abordajes, satisfacer las necesidades de los pacientes con enfermedad cardiovascular en los diferentes aspectos del tratamiento de la enfermedad.

\section{Recomendaciones}

Dado que la agrupación de los ítems resultante del análisis factorial exploratorio no coincide completamente con las dimensiones planteadas al inicio por el autor, se recomienda que investigaciones posteriores realicen un análisis factorial comprobatorio con el objetivo de seguir cualificando el instrumento.

Financiación: la investigación fue financiada por la Universidad Nacional de Colombia y por la Pontificia Universidad Javeriana.

Conflicto de intereses: ninguno declarado.

\section{Referencias}

1. World Health Organization. Global Status Report on noncommunicable diseases 2014. 2014 [visitado 2016 ago 25]; pp. 9-20. Disponible en http://apps.who.int/iris/bitstream/10665/148114/1/9789241564854_eng.pdf?ua=1

2. Mozaffarian D, Benjamin E, Go A, Arnett D, Blaha M, Cushman M et al. Heart Disease And Stroke Statistics - 2016. Update. A report from de American Heart Association. Circulation. 2016;133(8):000-000.

3. Departamento Administrativo Nacional de Estadística (DANE). Defunciones no fetales 2015, preliminar [visitado 2015 sep 30]. Disponible en http://www.dane.gov.co/index.php/esp/component/content/article/118-demograficas/estadisticas-vitales/6234-defunciones-no-fetales-2015-preliminar

4. Sandoval D, Chacón J, Muñoz R, Henríquez O, Koch E, Romero T. Influencia de factores psicosociales en la adherencia al tratamiento farmacológico antihipertensivo. Resultados de una cohorte del programa de salud cardiovascular de la región metropolitana de chile. Rev Med Chile. 2014;142(10):1245-52.

5. González Anglada MI. La adherencia, el talón de Aquiles de la enfermedad cardiovascular. Rev Calidad Asistencial . 2012;27(2):69-71.

6. Orozco Beltrán D, Carratala-Munuera C, Gil Guillen V. Mejorar la adherencia: una de las acciones más eficientes para aumentar la supervivencia de los pacientes en prevención secundaria. Rev Esp Cardiol. 2015;15(5):12-18.

7. Sanahuja M, Villagrasa V, Martínez Romero F. Adherencia terapéutica. Pharm Care Esp. 2012;14(4):162-7.

8. Martínez Domínguez G, Martínez Sánchez L, Lopera Valle J, Vargas Grisales N. La importancia de la adherencia terapéutica. Rev Venez Endocrinol Metab. 2016;14(2):107-16.

9. Pisano-González M, González-Pisano A. La modificación de los hábitos y la adherencia terapéutica, clave para el control de la enfermedad crónica. Enferm Clin. 2014;24(1):59-66.

10. Maldonado Reyes FJ, Vásquez Martínez VH, Loera Morales J, Ortega Padrón M. Prevalencia de adherencia terapéutica en pacientes hipertensos con el uso del cuestionario Martín Bayarre Grau. Aten Fam. 2016;23(2):48-52.

11. Zambrano R, Duitama J, Posada J, Flórez J. Percepción de la adherencia a tratamientos en pacientes con factores de riesgo cardiovascular. Rev Fac Nac Salud Pública. 2012;30(2):163-74.

12. Ortiz Suárez C. Instrumento para evaluar la adherencia en pacientes con factores de riesgo cardiovascular. Av Enferm. 2011;28(2):73-87.

13. Contreras Orozco A, Flórez Torres I, Herrera Lián A. Un instrumento para evaluar la adherencia: su validez facial y confiabilidad. Av Enferm. 2010;26(2):35-42.

14. Canales Vergara S, Barra Almagia E. Autoeficacia, apoyo social y adherencia al tratamiento en adultos con diabetes mellitus tipo II. Psicología y salud. 2014;24(2):167-73. 
15. Resnick B. The theory of self-efficacy. En Smith M, Liehr P, editors. Middle range theory for nursing. New York: Springer publishing company; 2014. p. 49-68.

16. Machado Alba J, Machado Duque M. Prevalencia de factores de riesgo cardiovascular en pacientes con dislipidemia afiliados al sistema de salud en Colombia. Rev Perú Med Exp Salud Pública. 2013;30(2):205-11.

17. Lamas C, Beloscar J, Restifo B, Gallardo A. Prevalencia de factores de riesgo cardiovascular en la ciudad de Funes (Santafé). Estudio Previene. Rev Fed Cardiol. 2016;45(1):34-9.

18. Organización Mundial de la Salud (OMS). Enfermedades cardiovasculares. Nota descriptiva [visitado2015 Jun 15]. Disponible en http://www.who.int/mediacentre/factsheets/fs317/es/

19. Soria-Trujano R, Vega-Valero CZ, Nava-Quiroz C. Escala de adherencia terapéutica para pacientes con enfermedades crónicas, basada en comportamientos explícitos. Altern Psicol. 2009;14(20):78-88.

20. Varela-Arevalo MT. El reto de evaluar la adherencia al tratamiento en la hipertensión arterial. Pensam Psicol. 2010;7(14):127-40

21. Skjong R, Wentworth B. Expert Judgement and risk perception. 2000 [visitado 2015 ene 15]. Disponible en http:// research.dnv.com/skj/Papers/SkjWen.pdf

22. Tristán A. Modificación al modelo de Lawshe para el dictamen cuantitativo de la validez de contenido de un instrumento objetivo. Av Medic. 2008;6:37-48.

23. Cerda J, Villarroel del P L. Evaluación de la concordancia inter-observador en investigación pediátrica: coeficiente de Kappa. Rev Chil Pediatr. 2008;79(1):54-8.

24. Campo A, Herazo E, Oviedo H. Análisis de factores: fundamentos para la evaluación de instrumentos de medición en salud mental. Rev Colomb Psiquiat. 2012;41(318):659-71.

25. Badia X, Lizán L. Estudios de calidad de vida. En Atención primaria. Conceptos, organización y práctica. Madrid: Elsevier; 2003.

26. Streiner D, Norman G, Cairney J. Scaling responses. En Streiner D, Norman G, Cairney J, editors. Health Measurement Scales, a practical guide to their development and use. New York: Oxford University Press; 2015. p. 38-65.

27. Pedrosa I, Suárez Álvarez J, García Cueto E. Evidencias sobre la validez de contenido: avances teóricos y métodos para su estimación. Acción Psicol. 2014;10(2):3-20.

28. Ortego Maté M. La adherencia al tratamiento. Variables implicadas. Educare21. 2004 [visitado 2015 ene 18];8. Disponible en http://enfermeria21.com/educare/educare08/ensenando/ensenando2.html

29. Silva G, Galeano E, Correa JO. Adherencia al tratamiento, implicaciones de la no adherencia. Acta Med Colomb. 2005;30(4):268-73.

30. Veliz-Rojasa L, Mendoza-Parrab S, Barrigac OA. Adherencia terapéutica y control de los factores de riesgo cardiovasculares en usuarios de atención primaria. Enferm univ. 2015;12(1):3-11.

31. Martín Alfonso L, Grau Ábalo J, Espinosa Brito D. Marco conceptual para la evaluación y mejora de la adherencia a los tratamientos médicos en enfermedades crónicas. Rev Cub Salud Pública. 2014;40(2):225-38.

32. Barrera Ortiz L, Campos de Aldana ME, Carreño SP, Carrillo GM, Chaparro Díaz L, Jaimes L et al. Validez de constructo y confiabilidad del instrumento de soporte social en la enfermedad crónica. Rev Cult Cuidado. 2013;10(2):8-19.

33. Olivari-Medina C, Urra-Medina E. Autoeficacia y conductas de salud. Cienc enferm. 2007;XIII(1):9-15.

34. Reigal R, Videra A. Frecuencia de práctica física y autoeficacia en la tercera edad. Rev Int Med Cienc Act Fis Deporte. 2013;13(49):107-20.

35. Berciano S, Ordovás J. Nutrición y salud cardiovascular. Rev Esp Cardiol. 2014;67(9):738-47.

36. Perk J, De Backer G, Gohlke H, Graham I, Reiner Z, Verschuren M. Guía europea sobre prevención de la enfermedad cardiovascular en la práctica clínica. Rev Esp Cardiol. 2012;65(10):937.e1-e66.

37. Nunally J. Psychometric Theory. New York: McGraw-Hill; 1978.

38. Carvajal A, Centeno C, Watson R, Martínez M, Sanz A. ¿Cómo validar un instrumento de medida de la salud? Anales Sis San Navarra. 2011;34(1):63-71. 